\title{
Breakthrough in breast reconstruction in the context of COVID-19: safety and efficiency of endoscopic breast reconstruction at a day surgery center
}

\author{
Jiao Zhou\#, Xinran Liu\#, Yu Feng, Juan Li, Xiangquan Qin, Yixuan Huang, Huanzuo Yang, Mengxue Qiu, \\ Yang Liu, Hongsheng Ma, Qing Lv, Zhenggui Du \\ Department of Breast Surgery, West China Hospital, Sichuan University, Chengdu, China \\ Contributions: (I) Conception and design: Z Du; (II) Administrative support: Q Lv; (III) Provision of study materials or patients: Z Du; (IV) Collection \\ and assembly of data: J Zhou, X Liu; (V) Data analysis and interpretation: J Zhou; (VI) Manuscript writing: All authors; (VII) Final approval of \\ manuscript: All authors. \\ \#These authors contributed equally to this work. \\ Correspondence to: Zhenggui Du. Department of Breast Surgery, West China Hospital, Sichuan University, Chengdu 610041, China. \\ Email: docduzg@163.com.
}

Background In recently years, breast endoscopic reconstruction surgery is becoming increasingly popular. And we have explored a series of endoscopic breast reconstruction procedures and applied it to our day surgery under the epidemic control of the novel coronavirus.

Methods: The present study was a retrospective analysis. Patients who underwent unilateral breast endoscopic reconstruction surgery in the West China Hospital from April 2017 to February 2021 were included in the study. Patients were divided into the following three groups: ward exploration period (WEP), ward maturation period (WMP), and day surgery period (DSP), respectively. We compared the results of postoperative complications, hospitalization costs, operation time, and BREAST-Q (a patient-reported outcome instrument measuring health-related quality-of-life and patient satisfaction in breast surgery) scale scores among the three groups of patients.

Results: A total of 66 patients were included (WEP n=30, WMP n=14, DSP n=22). Four people refused to complete the BREAST-Q scale, and five patients missed complication record sheets. Patients in the DSP and WMP groups had slightly higher postoperative satisfaction with their breasts than WEP, but there was no statistically significant difference ( 3 months postoperatively: WEP $v s$. WMP $=0.515, \mathrm{WEP} v s$. DSP $=0.418$, WMP vs. DSP =0.982). On the postoperative BREAST-Q scale scores of psychosocial, sexual life and chest well-being, patients with DSP scored slightly higher than those with WEP versus WMP, but there was no statistically significant difference. The incidence of postoperative complications was generally higher in the WEP group than in the WMP and DSP groups, but there was no statistically significant incidence of either major or minor complications $(\mathrm{P}=1.000)$. With the use of prostheses and mesh, patients in the DSP group had lower hospitalization costs than other two groups. In terms of operative time, patients in the WMP and DSP groups had shorter operative times compared with the WEP group, and the results were statistically significant (WEP vs. WMP =0.000, WEP vs. DSP =0.000, WMP vs. DSP =0.243).

Conclusions: We believe that performing our newly developed endoscopic breast reconstructive surgery at a day surgery center is safe and reliable.

Keywords: Day surgery; endoscopy; inverse sequence method; Huaxi hole 1; breast reconstruction

Submitted Jun 01, 2021. Accepted for publication Aug 05, 2021.

doi: $10.21037 / g s-21-405$

View this article at: https://dx.doi.org/10.21037/gs-21-405 


\section{Introduction}

To minimize surgical trauma and promote recovery, breast cancer surgery has evolved from radical surgery and extended radical surgery to modified radical surgery and breast-conserving surgery (1-4). However, for patients with more diffuse lesions, breast-conserving surgery is less safe, but the loss of the breast can also be very psychologically taxing for the patient (5-7). Breast reconstruction surgery is becoming an increasingly popular alternative. In December 2019, coronavirus 2019 (COVID-19) was discovered and the virus has since become a widespread pandemic, with a high transmission rate and pathogenicity. Studies have shown that controlling staff turnover in public has a significant effect on controlling the spread of COVID-19 $(8,9)$. Therefore, in order to control the spread of the COVID-19, a number of countries, including China, Italy, and the UK have taken measures to minimize the movement of people. As major public places, many hospitals are reducing the length of stay of patients to reduce the risk of disease transmission. As a result, day surgery was prevalent in domestic during this time. However, for patients who need breast reconstruction, surgery is difficult and there are many postoperative complications $(10,11)$, so traditional breast reconstruction patients need to be hospitalized for postoperative observation and treatment. However, this makes it more difficult to prevent and control epidemic in hospitals. Therefore, it is important to find a safe and effective method of breast reconstruction surgery that can be performed in ambulatory surgery centers, therefore reducing the length of stay of patients.

When Philippe Mouret performed the first laparoscopic cholecystectomy in 1987, the era of minimally invasive surgery began, and gradually gained popularity. In recent years, with the development of surgical techniques, endoscopic kidney extraction and the radical treatment of gastrointestinal tumors are also gradually being performed worldwide. However, breast endoscopic reconstruction surgery is limited worldwide due to the lack of natural lumens in the breast. Therefore, more breast specialists devote themselves to explore breast endoscopic reconstruction surgery in recent years. The most popular methods of breast endoscopic reconstruction are roboticassisted breast endoscopic reconstruction and lipolysis breast endoscopic reconstruction. However, these two surgical procedures cannot be used in ambulatory surgery centers due to the high equipment requirements, high cost, and long operation time $(12,13)$. Therefore, it is important to explore breast endoscopic reconstructive surgery modalities suitable for daytime surgery to meet the needs of epidemic prevention and control.

Over the past 3 years our hospital has conducted a series of explorations into breast endoscopic reconstructive surgery. Under the epidemic, we proposed new concepts, such as the inverse sequence method and small areolar incision (Huaxi hole 1) and introduced them into breast endoscopic reconstructive surgery, resulting in significantly shorter operation times, low postoperative complication rates, and high patient satisfaction. To shorten the patient's hospital stay and reduce epidemic transmission, we applied this procedure to our day surgery center and found that breast endoscopic reconstructive at our day surgery center was safe and reliable during the follow-up of complications and the BREAST-Q scale. The aim of the present study was to compare patients in the ward exploration period (WEP), the ward mature period (WMP), and the day surgery period (DSP) in terms breast endoscopic reconstructive surgery in our hospital to present the experience of managing day breast endoscopic reconstructive surgery and to explore the safety and effectiveness of performing daytime mature breast endoscopic reconstructive surgery. We present the following article in accordance with the STROBE reporting checklist (available at https://dx.doi. org/10.21037/gs-21-405).

\section{Methods}

All procedures performed in this study involving human participants were in accordance with the Declaration of Helsinki (as revised in 2013). The study was approved by the ethics board of West China Hospital of Sichuan University (No. 2021-863). Individual consent for this retrospective analysis was waived.

\section{Patients}

The present study was a retrospective analysis. Patients who underwent unilateral breast endoscopic implant-based reconstructive surgery from April 2017 to February 2021 at the Department of Breast Surgery, West China Hospital, were included in the study. Patients were divided in the WEP, WMP, and DSP groups (WEP refered to surgery performed on the ward and the procedure was immature. WMP refered to surgery performed on the ward and the procedure was mature. DSP refered to surgery performed in an ambulatory surgery center and the procedure was 
mature). The number of cases in this institution during the study period determined the sample size.

Inclusion criteria for ambulatory breast endoscopic reconstruction surgery included oncologic criteria and ambulatory surgery criteria. Oncological criteria: were as follows: (I) pathological diagnosis of breast cancer and tumor $<5 \mathrm{~cm}$; (II) tumor was a multicentric lesion and breast-conserving surgery could not be performed; and (III) patient had certain cosmetic requirements for breast reconstruction. Ambulatory surgery criteria were as follows: (I) age 18-70 years with no serious complications or functional organ disorders; (II) regular postoperative follow up. Patients were able to undergo surgery at an ambulatory surgery center; (III) body mass index $\leq 35 \mathrm{~kg} / \mathrm{m}^{2}$; (IV) American Society of Anesthesiologists (ASA) score $\leq$ II; and (V) day patients should be accompanied by an adult after discharge and had an address close to the hospital.

\section{Preoperative preparation}

At the outpatient clinic, the surgeon introduced the procedure and discusses risks with patients who meet the standard of endoscopic breast reconstruction. If the patient chose to have the endoscopic breast reconstruction performed in the ward, patients would go to the admission service center for admission-related procedures and complete preoperative preparations with the help of doctors and nurses.

If the patient chose to have endoscopic breast reconstruction performed at the day surgery center, the surgeon would issue a admission certificate and perform relevant preoperative examinations. The patient was required to complete the preoperative examination 1 month before the operation to ensure that the results of the examination were accurate and reliable. The patient presented at the anesthesia clinic 3 days before the operation to assess the risk of anesthesia and signed the relevant documents. In the afternoon, the patients attended the surgeon's office for breast measurement and to determine the type and size of the prosthesis, and signed the relevant documents. After completing the examination and signing the documents, the patient took the relevant information to the day surgery center to make an appointment for the operation. If a problem was found during the appointment, the doctor of the day surgery center would contact the surgeon in time to deal with it. If there was no problem, the surgeon and the patient would be informed of the specific operation time. On the day before the operation, the day surgery center doctor would call the patient again to approve the operation time and make preoperation education before operation. When the patient was admitted to hospital on the morning of the operation, the ward nurse would recheck the preoperative examinations and make preoperative education again. The surgeon would also make surgical markers of patients before operation, arranged the medicine for surgery, and if sentinel lymph node biopsy (SLNB) was required, would contact a physician in a nuclear medicine department to inject nuclides to trace sentinel lymph nodes.

\section{Surgical modalities}

\section{WEP}

The patient was placed in a lying position with the affected upper limb abducted. A 5-6-cm axillary incision was made. SLNB or axillary lymph node dissection (ALND) was performed as needed under direct vision. A 3-5-cm operating area was freed using Peng's multifunctional operative dissector (PMOD) (Shuyou Surgical, Hangzhou, China) in the retromammary space. The disposable wound protector (herein referred to as the "protector") (Surkon Medical, Wuxi, China), wrapped by the opening end of a sterile surgical glove (7\#), was placed through the incision. Three trocars $(5.5,5.5$, and 12.5 mm; Aesculap Inc., Center Valley, USA) was placed via the fingertips of the glove and charged with $\mathrm{CO}_{2}$ $(12 \mathrm{mmHg}$ ) to maintain sufficient optical cavity tension.

Patients using prosthesis undergone total subpectoral reconstruction. In the early stage, the subcutaneous layer of breast (by PMOD), retromammary space (by coagulation hook), and subpectoral space (by coagulation hook) were dissected in turn (sequential method). In the mid-term, retromammary space (by coagulation hook), subcutaneous layer of breast (by PMOD), and subpectoral space (by coagulation hook) were dissected in turn. At the final stage, subpectoral space (by coagulation hook), retromammary space (by coagulation hook), and subcutaneous layer of breast (by PMOD) were dissected in turn (inverse order method). The PMOD is placed via Huaxi hole 1 (located next to the areola in the upper-outer quadrant) when dissecting the subcutaneous layer of breast. Then, after irrigating the implant cavity, the prosthesis was placed behind the pectoralis muscle and the incision was closed after placement of the drainage tube. If the implant was an expander, the expander was placed behind the pectoral muscle and then the appropriate amount of $0.9 \% \mathrm{NS}$ was injected into the 
expander according to the size of the patient's breast

Patients who using prosthesis and mesh undergone semi-subpectoral reconstruction. The lower edge of the pectoralis muscle was cut when dissecting the subpectoral space. The mesh was placed prior to the placement of the prosthesis using a traction wire parachute mesh method, and the mesh was secured to the surrounding tissue. Finally, the prosthesis was placed.

\section{WMP and DSP}

Both the WMP and DSP groups underwent operation by the inverse sequence method and the Huaxi hole 1. The traction wire parachute mesh method was used to place the mesh. The surgical procedure was detailed in Video 1.

\section{Postoperative management}

Patients in the WEP and WMP groups were discharged after 3 days of observation. In the DSP group, patients were discharged $24 \mathrm{~h}$ after admission, and postoperative education were given by nurses and doctors at the day surgery center. For all patients, the drainage tube was removed when the drainage fluid is less than $30 \mathrm{~mL}$ for 3 consecutive days and there was no bleeding. An elastic corset was worn for 4 weeks after $48-72$ h postoperatively. To drop the implant, began squeezing the breast inward and downward and pushing on the implant 3-5 days after surgery. To reduce edema and prevent thrombosis, patients were asked to abduct the affected upper arm about 1 week after surgery.

Because the duration of hospital observation was shorter in the DSP group compared with the other two groups, closer follow up was performed in addition to postoperative nursing education for patients and their family in the DSP group to reduce bias in recording complications and to ensure the safety of patients after discharge. Patients in the WEP and WMP groups were asked to visit the clinic at 1 week, 1 month, and 2 months after discharge. In the DSP groups, patients were asked to visit the clinic for follow up at 4 and 15 days after surgery. Doctors in the day surgery center followed up patients by telephone at $1-3,7,30$, and 60 postoperatively. In May 2021, the difference in BREAST-Q (version 2.0) scores between 1 month postoperatively, 3 months postoperatively, and preoperatively were counted separately by questionnaire distribution. BREAST-Q outcome measures were calculated using the Q-Score Scoring Software package.

In addition, postoperative complications were evaluated and documented by doctors at the time of follow up. Patients were followed up at least once a year at the outpatient clinic by telephone. The endpoint we set for follow up was patient death. The cut-off time for follow up of complications in this study was May 2021. Major complications were defined as events related to the reconstruction that required additional surgical intervention. These included bleeding, surgical site infection, wound dehiscence, implant rupture, and implant explantation. Surgical site infections were considered to be ineffective with oral and intravenous antibiotics and required surgical intervention. Bleeding as considered to be ineffective to stop bleeding by compression, requiring surgical intervention to stop. Minor complications were defined as events that could be treated conservatively, not requiring surgical intervention. The majority of these included surgical site infection that responded to an adequate course of either oral or intravenous antibiotics and bleeding that can be stopped by compression. In addition, fluid accumulation after extubation were considered to require drawing with an empty needle or repositioning of the drainage tube.

\section{Statistical analysis}

Data were analyzed using descriptive statistics, such as means, ranges, standard deviations, and proportions. Categorical data were presented as percentages. Among the statistics for the incidence of complications, the incidence was counted in this study after removing the lost interviewers. Continuous variables were compared using unpaired Student's $t$-test. Rates were analyzed using $\chi^{2}$-tests. Graphing was done using $\mathrm{R}$ for Windows (version 3.6.2, $\mathrm{R}$ Development Core Team 2020). All statistical evaluations were performed using the SPSS 25 for Windows (SPSS Inc., Chicago, IL). Results with $\mathrm{P}<0.05$ were considered statistically significant.

\section{Results}

A total of 66 patients underwent endoscopic reconstruction operation, with 30 in the WEP, 14 in the WMP, and 22 in the DSP. In the process of completing the BREAST-Q scale, 3 people and 1 person in the WEP and WMP groups, respectively, refused to complete the questionnaire. During the complication count, 4 people and 1 person in the WEP and WMP groups, respectively, were unable to find a complication record sheet. Flowchart of data selection was detailed in Figure 1. 


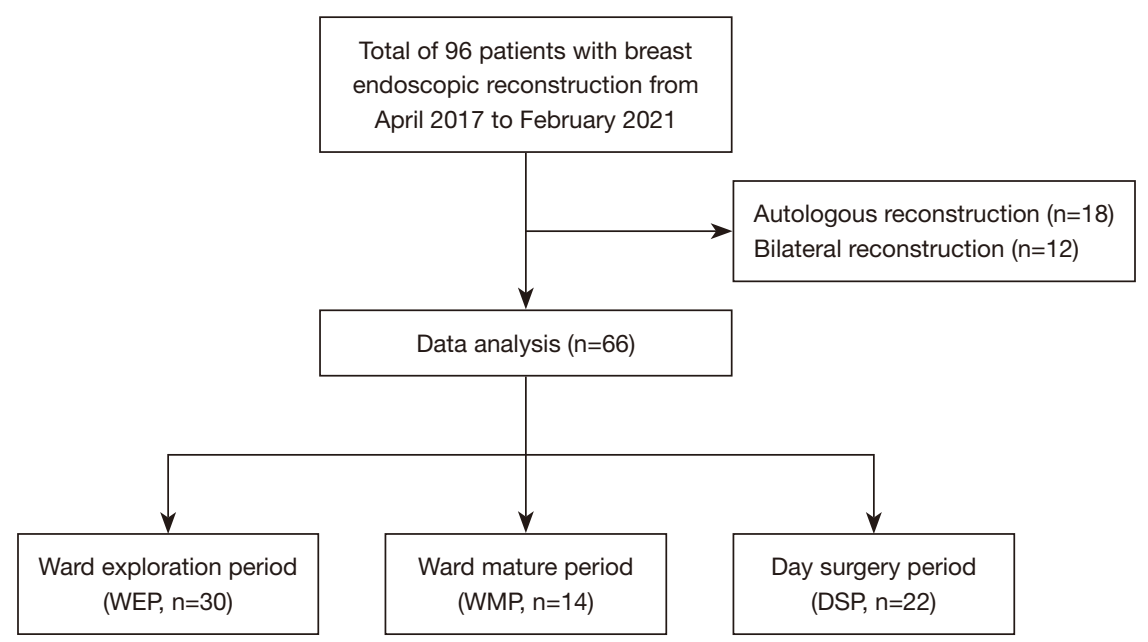

Figure 1 Flowchart of data selection. WEP, ward exploration period; WMP, ward maturation period; DSP, day surgery period.

\section{Patient characteristics}

All three groups had predominantly invasive cancer and none of the axillary lymph node metastases exceeded N3. In addition, the proportion of patients with MBI (Body Mass Index) greater than 24 did not exceed $15 \%$ in any of the three groups, and the maximum diameter of the mass did not exceed $5 \mathrm{~cm}$ in all patients. When the three groups were compared, only the comparison of implant type was significantly different $(\mathrm{P}=0.031)$; the rest were not significantly different. The different characteristics of the three groups were presented in Table 1 .

\section{Esthetic evaluation}

All patients had only a 5-6-cm axillary incision and a small 5 -mm areolar incision (Huaxi hole 1). After a short period of recovery, the scar of the small areola incision gradually faded, so the overall esthetics of the breast was good and the patient's satisfaction was high. The reconstruction effect of the 3 periods was shown in Figure 2.

In the present study, the BREAST-Q scale was used to assess patients' postoperative esthetic score. All three groups had significantly lower scores at 1 month postoperatively compared with preoperative scores, but these improved at 3 months postoperatively. Among the three groups, postoperative breast satisfaction scores were slightly higher in the WMP and DSP groups than in the WEP group, but were not statistically significant ( 3 months postoperatively: WEP $v s$. WMP $=0.515$, WEP $v$. $\mathrm{DSP}=0.418, \mathrm{WMP} v s . \mathrm{DSP}=0.982)$. Each patient's breast satisfaction score and the changes in the difference between preoperatively and 1 and 3 months postoperatively for the 3 periods were shown in Figure 3.

\section{Operation time and cost}

The mean total hospital costs for all three groups ranged from 44,000 to 46,000 yuan. However, there was a significant difference in hospitalization costs due to different implants. The number of patients using various implants in the 3 periods and the differences in hospitalization costs for the 3 periods with different implants were shown in Figure 4.

Patients in the WEP $(313.43 \pm 48.923 \mathrm{~min})$ group had a significantly longer operative time than those in the WMP $(203.79 \pm 28.631 \mathrm{~min})$ and DSP $(188.59 \pm 21.429 \mathrm{~min})$ groups, and the results were statistically significant (WEP vs. WMP $\mathrm{P}=0.000$, WEP $v s$. DSP $\mathrm{P}=0.000$, WMP vs. DSP $\mathrm{P}=0.243$ ). The operative times of the 3 periods were shown in Figure 5 .

\section{Postoperative complications}

Mean follow-up months for complications in the WEP, WMP, and DSP groups were 20.43 (range, 9-48), 6.07 (range, 3-9), and 5.23 (range, 3-7), respectively. In the comparison of postoperative complications among the three groups of patients, the incidence of complications was generally higher in the WEP group than in the WMP and DSP groups, but the incidence of both major and minor complications was not statistically significant $(\mathrm{P}=1.000)$. The details of postoperative complications were shown in Table 2. 
Table 1 Patient characteristics

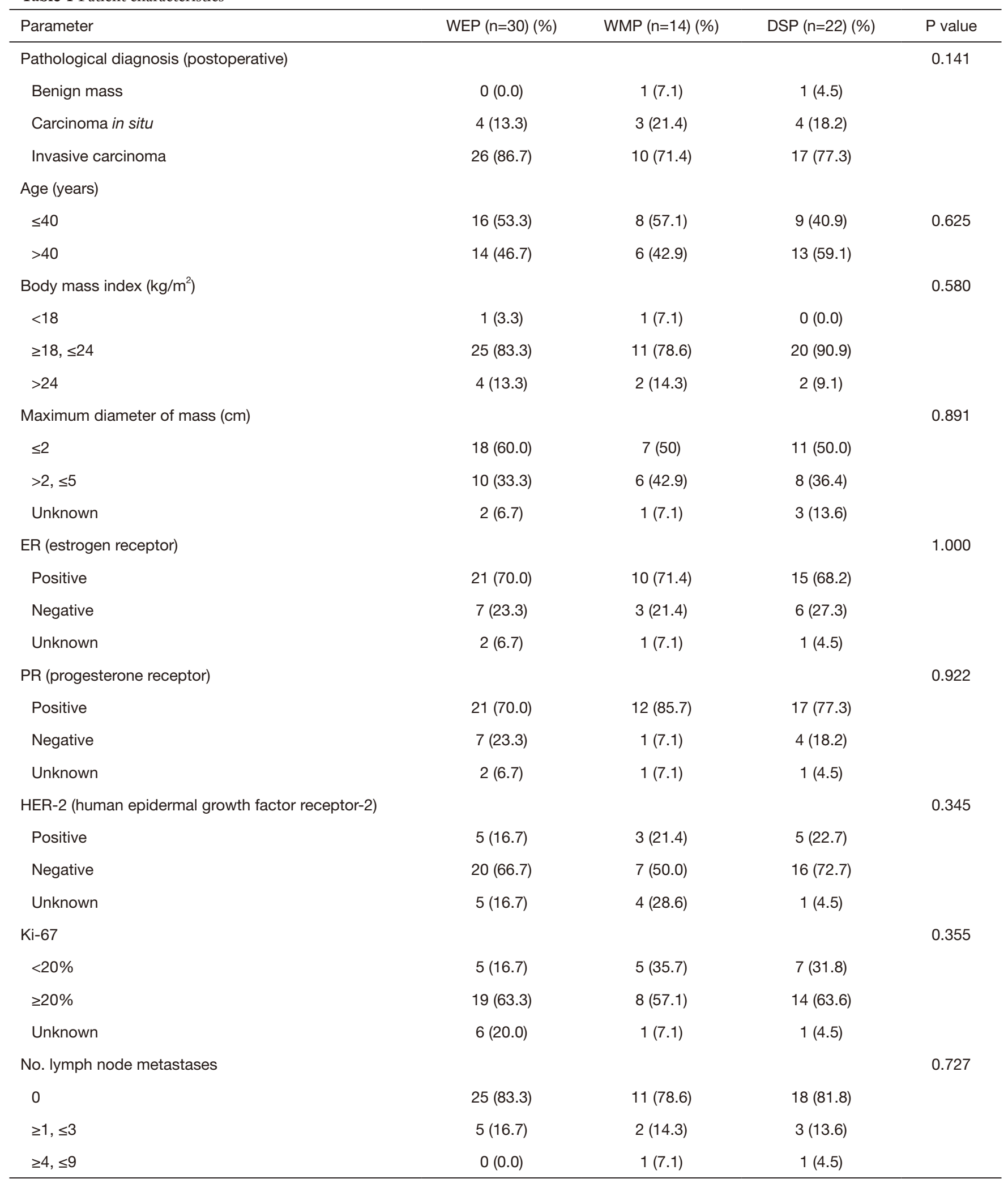

Table 1 (continued) 
Table 1 (continued)

\begin{tabular}{lccc}
\hline Parameter & WEP $(\mathrm{n}=30)(\%)$ & WMP $(\mathrm{n}=14)(\%)$ & DSP $(\mathrm{n}=22)(\%)$ \\
\hline Axillary surgery & & & \\
No axillary surgery & $0(0.0)$ & $1(7.1)$ & $1(4.5)$ \\
Sentinel lymph node biopsy & $22(73.3)$ & $10(71.5)$ & $16(72.7)$ \\
Lymph node dissection & $8(26.7)$ & $3(21.4)$ & $5(22.7)$ \\
Implant & & $1(7.1)$ & 0.031 \\
Expander & $4(13.3)$ & $10(71.4)$ & $7(31.8)$ \\
Prosthesis & $12(40.0)$ & $3(21.4)$ & $15(68.2)$ \\
Prosthesis and mesh & $14(46.7)$ & & 0.031 \\
\hline
\end{tabular}

DSP, day surgery period; WEP, ward exploration period; WMP, ward maturation period.
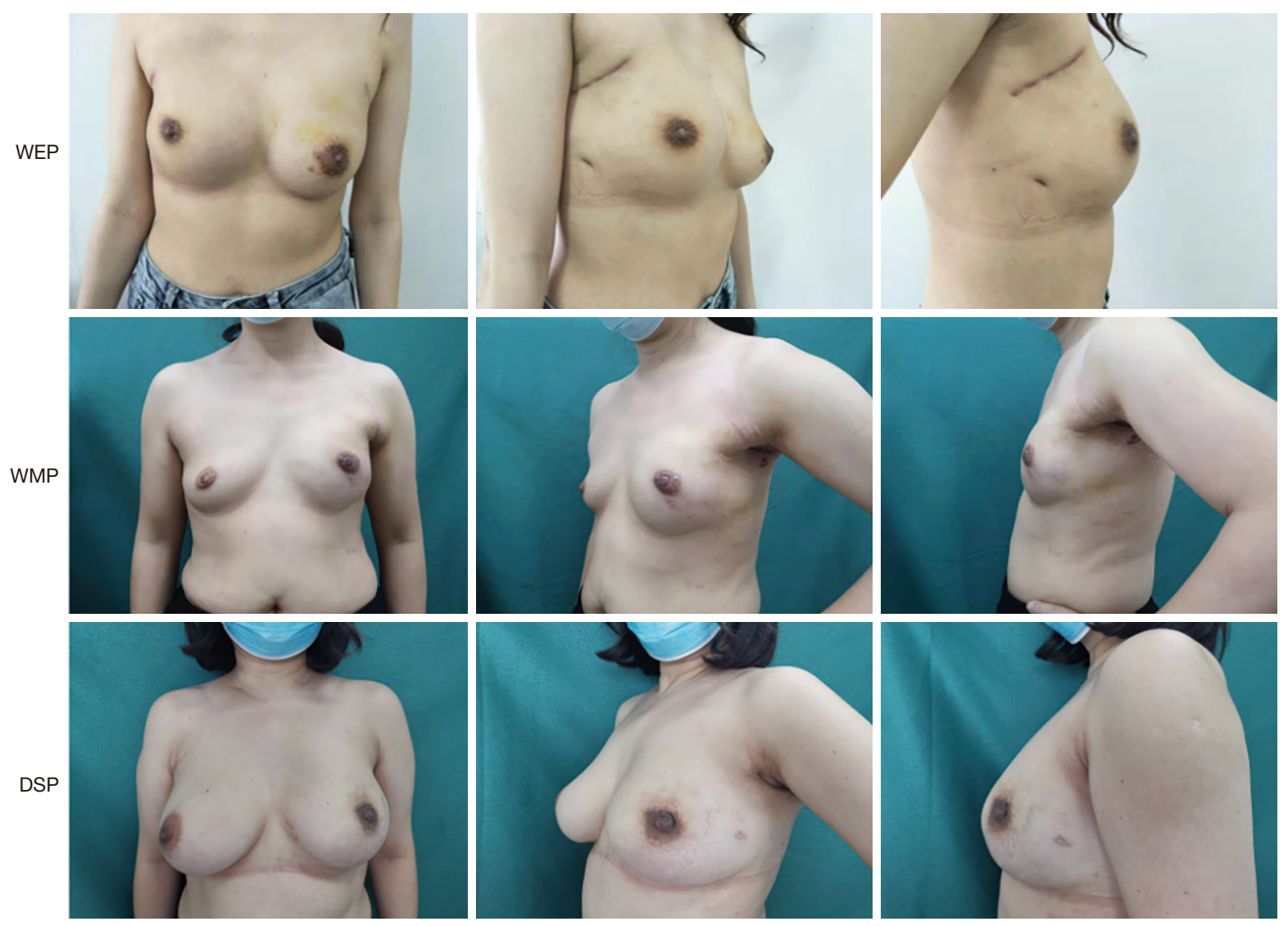

Figure 2 Orthopantomogram, oblique lateral and lateral body results of endoscopic breast reconstruction patients in 3 periods at 3 months postoperatively. DSP, day surgery period; WEP, ward exploration period; WMP, ward maturation period. 
A

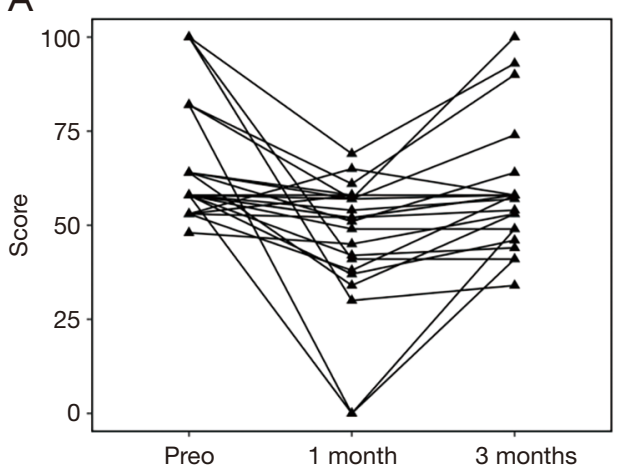

C

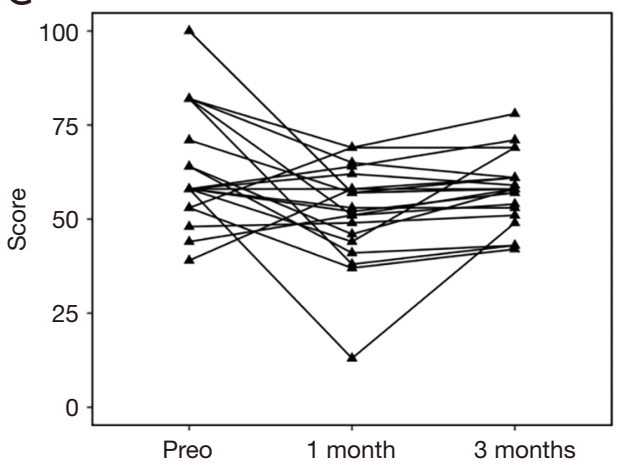

B

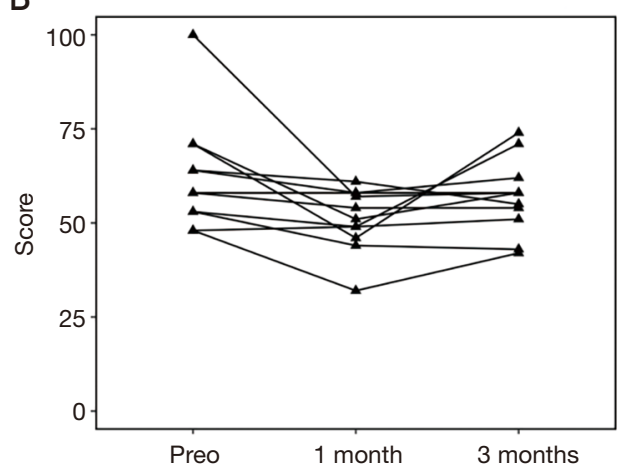

D

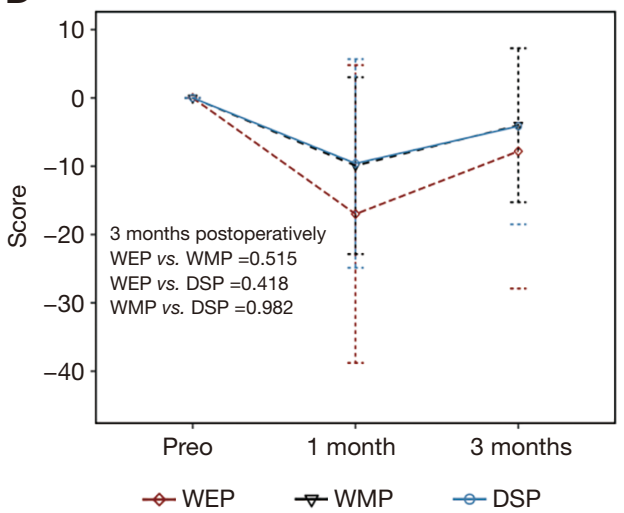

Figure 3 Preoperative and 1-month and 3-month postoperative breast satisfaction scores and changes in mean breast satisfaction scores of the three groups of patients. Preoperative and postoperative breast satisfaction scores of patients in the WEP (A), WMP (B), and DSP (C) groups, respectively. (D) Mean change between postoperative and preoperative breast satisfaction for the three groups of patients. WEP, ward exploration period; WMP, ward maturation period; DSP, day surgery period.

\section{Functional evaluation}

Psychosocial, sexual and chest well-being components of the BREAST-Q scale were used to assess patients' postoperative functional scores. The scores of all three groups were significantly lower in the first postoperative month compared with the preoperative period, but improved in the third postoperative month. However, among the three groups, patients in the DSP group had slightly higher postoperative scores than those in the WEP and WMP groups, but there was no statistically significant difference. The changes in the difference between the preoperative and 1 and 3 months after the operation values of psychosocial, sexual, and chest well-being in the 3 periods were shown in Figure 6.

\section{Short-term oncological safety}

Due to the short follow-up time, the long-term local and systemic complications were not observed in the present study, but there were no local recurrence and systemic metastasis in any of the patients 3 months after operation.

\section{Discussion}

During our 3-year exploration of breast endoscopic reconstruction surgery, we developed the inverse sequence method and the Huaxi hole 1. With their application, the operation time of endoscopic breast reconstruction surgery was significantly shortened. Due to COVID-19, we successfully applied this surgical method to our ambulatory surgery center to reduce staff turnover and strengthen epidemic prevention and control. Comparison of postoperative complications and BREAST-Q scores during the WEP, WMP, and DSP, we found there was no significant difference between all three groups of patients. Therefore, we believe it is safe and reliable to perform 

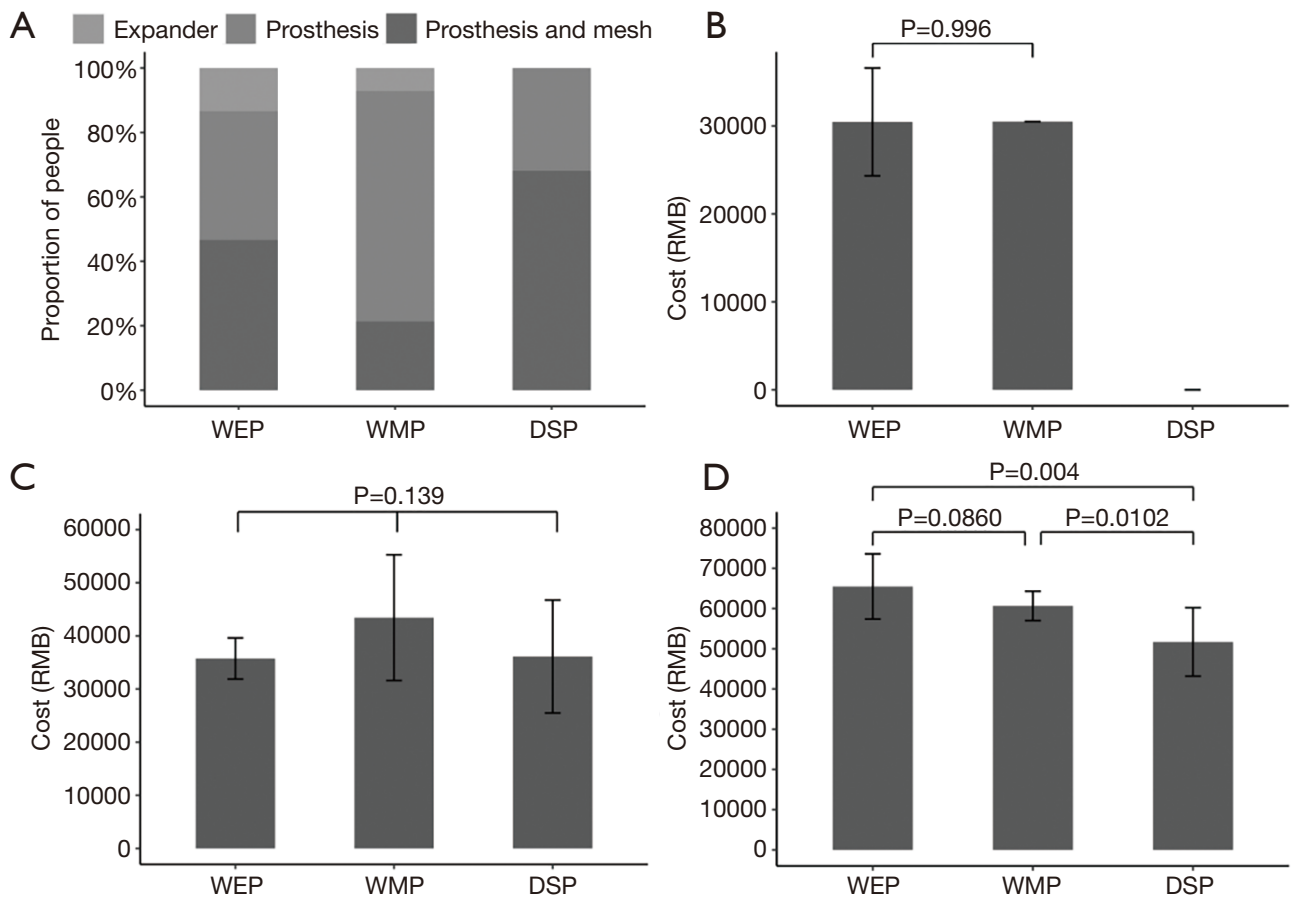

Figure 4 The number of patients using various implants in the 3 periods and the differences in hospitalization costs for the 3 periods with different implants. (A) Use of different implants in the three groups of patients. Average hospitalization costs for the three groups of patients with expander alone (B), prostheses alone (C), and prostheses and mesh (D), respectively. DSP, day surgery period; WEP, ward exploration period; WMP, ward maturation period.

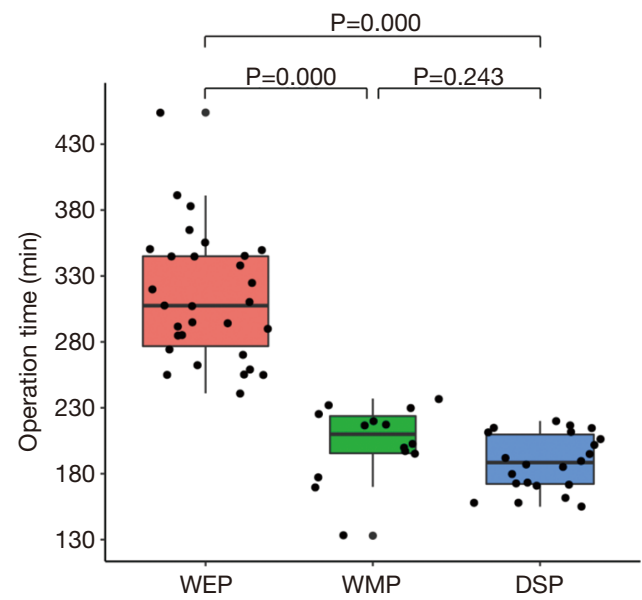

Figure 5 Distribution of surgery times for the three groups of patients. DSP, day surgery period; WEP, ward exploration period; WMP, ward maturation period.

breast endoscopic reconstructive surgery in an ambulatory surgery center when the technology was mature (the improved procedure was proven to be safe and effective after being performed in the inpatient unit).

Compared with previously published studies, which reported a $20-30 \%$ complication rate after open prosthetic reconstruction (14-16), all three groups of patients had a lower incidence of major postoperative complications (WEP vs. WMP vs. DSP: $3.8 \%$ vs. $0.0 \%$ vs. $0.0 \%$ ) and $<20 \%$ of minor complications, which may be attributed to the use of endoscopy instruments. During endoscopy, the smaller surgical incision and the corresponding reduction in the directly exposed area, coupled with the establishment of the air cavity allowing repeated airflow flushing within the incision, reduces the possibility of bacterial colonization, resulting in a significant reduction in the incidence of postoperative infections and other complications. In addition, the use of the endoscopy allows for a less invasive procedure, resulting in relatively less postoperative lymphedema in the affected arm and less fluid accumulation after extubation. However, there were no significant differences between the three groups in terms of major complications and minor complications. This also further confirms that surgery at ambulatory surgery centers is safe 
Table 2 Postoperative complications

\begin{tabular}{|c|c|c|c|c|}
\hline Parameter & WEP $(n=30)(\%)$ & WMP $(n=14)(\%)$ & DSP $(n=22)(\%)$ & $P$ value \\
\hline \multicolumn{5}{|l|}{ Major complications } \\
\hline Bleeding & $0(0.0)$ & $0(0.0)$ & $0(0.0)$ & \\
\hline Surgical site infection & $1(3.8)$ & $0(0.0)$ & $0(0.0)$ & 1.000 \\
\hline Implant rupture & $0(0)$ & $0(0.0)$ & $0(0.0)$ & \\
\hline Implant explantation & $1(3.8)$ & $0(0.0)$ & $0(0.0)$ & 1.000 \\
\hline Total $^{*}$ & $1(3.8)$ & $0(0.0)$ & $0(0.0)$ & 1.000 \\
\hline \multicolumn{5}{|l|}{ Minor complications } \\
\hline Mild flap ischemia & $2(7.7)$ & $1(7.7)$ & $1(4.5)$ & 1.000 \\
\hline Lymphedema of surgical side & $4(15.4)$ & $2(15.4)$ & $2(9.0)$ & 0.792 \\
\hline Subcutaneous effusion post-extubation & $3(11.5)$ & $0(0.0)$ & $1(4.5)$ & 0.528 \\
\hline Incision complications & $0(0.0)$ & $0(0.0)$ & $1(4.5)$ & 0.574 \\
\hline Capsular contracture & $0(0.0)$ & $0(0.0)$ & $0(0.0)$ & \\
\hline Total $^{*}$ & $5(19.2)$ & $2(15.4)$ & $4(18.2)$ & 1.000 \\
\hline Venous thromboembolism & $0(0.0)$ & $0(0.0)$ & $0(0.0)$ & \\
\hline Cardiopulmonary complication & $0(0.0)$ & $0(0.0)$ & $0(0.0)$ & \\
\hline
\end{tabular}

*, breasts with $\geq 1$ complication were computed once. DSP, day surgery period; WEP, ward exploration period; WMP, ward maturation period.

and reliable when the technology is mature $(17,18)$.

In terms of postoperative esthetics, compared to some reported postoperative satisfaction scores of 57-59 for open reconstruction in patients with a follow up of more than 1 year $(19,20)$, all three groups of patients in the present study had higher satisfaction at 3 months postoperatively $($ WEP vs WMP vs DSP = 57.38 vs. 57.84 vs. 60.77), and we believe that with gradual recovery and scar fading, postoperative breast satisfaction will further improve. Similarly, the surgical scar with robot-assisted reconstruction was also more often located in the axilla, which was not easily detected, and breast satisfaction was higher than with open reconstruction (21). Therefore, we believe it is important to hide the surgical scar and maintain the overall esthetics of the breast in breast reconstruction surgery. In addition, in this study, patient satisfaction was significantly lower in the postoperative WEP group than in the WMP and DSP groups. This was because, in the
WEP group, due to the selection of incision location was not experienced, and in order to ensure the safety of the surgery as much as possible, the axillary incision was mostly outward and upward. The incision was obviously exposed during upper limb abduction and lifting, making the overall esthetics of the breast slightly less attractive.

Although there was no significant difference in postoperative functional scores between the 3 periods, patient satisfaction was slightly higher in the DSP than in the WEP and WMP, which was related to the more mature surgical technique and the fact that patients received more care and support after surgery (22). In terms of sexual life, since there was no significant difference in age between the three groups, we believe that the higher satisfaction of patients in the DSP group is related to the careful and scientific companionship of their sexual partners who received relevant nursing education after returning home (23).

The procedure time was significantly shorter in the 

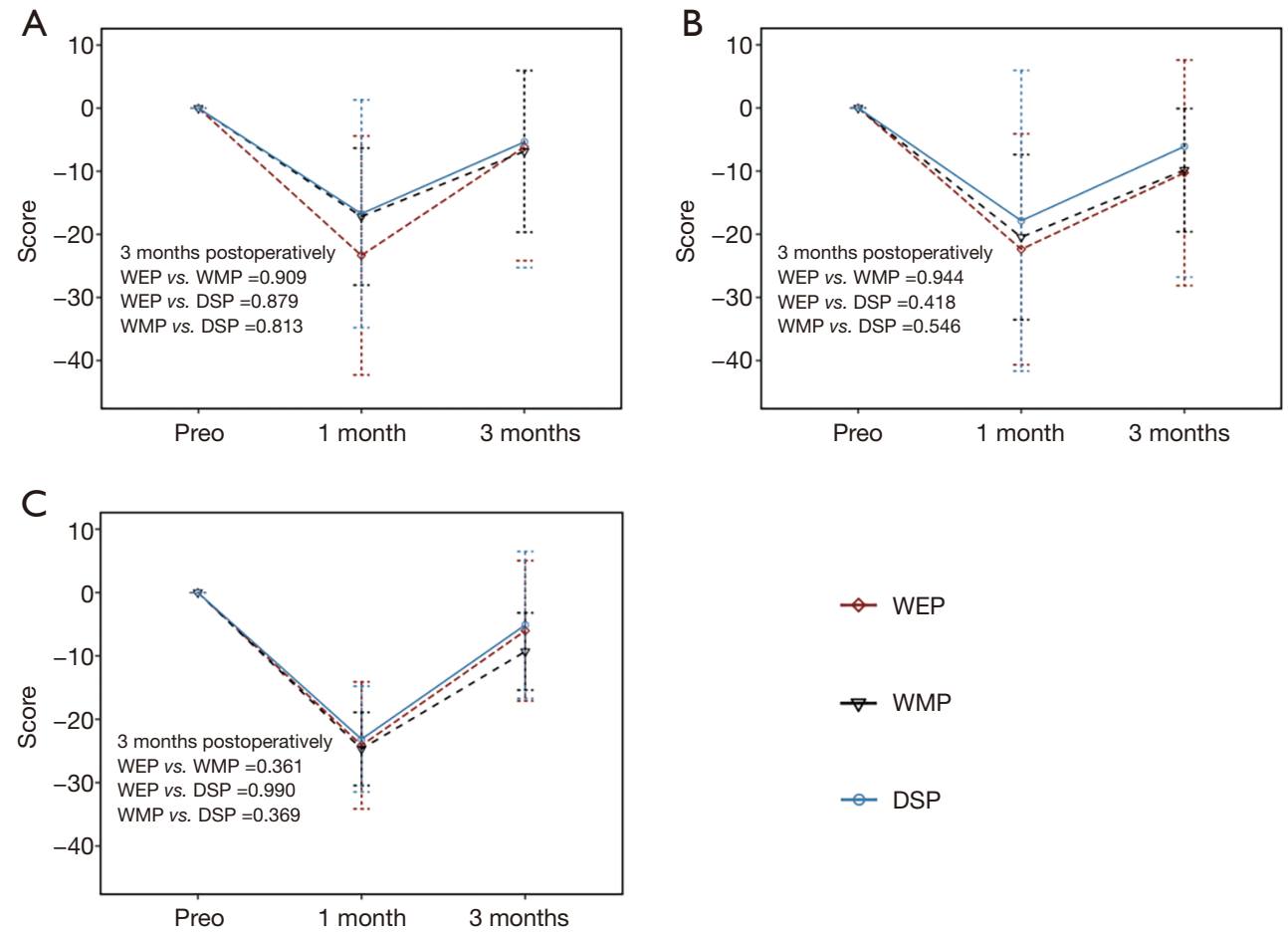

Figure 6 Changes in the mean scores of functional scores in the three groups of patients. Changes in the mean BREAST-Q (a widely used patient-reported outcome instrument measuring health-related quality-of-life and patient satisfaction in breast surgery) scores of the three groups of patients before and after surgery in the 3 aspects of psychosocial (A), sexual (B) and chest well-being (C), respectively. DSP, day surgery period; WEP, ward exploration period; WMP, ward maturation period.

WMP and DSP groups compared with the WEP group (313.55 vs. 203.79 vs. $188.59 \mathrm{~min}$, respectively), which was closely related to the use of the inverse sequence method and the Huaxi hole 1. The operative time for partial endoscopic breast reconstruction using the sequential method was approximately $340 \mathrm{~min}(240-442 \mathrm{~min})(24)$, which was much higher than the time for the reverse sequence method in the present study. During the WEP, we found that in the process of using the sequential method, the front layer of the tissue gap was propped open by the air cavity and the effect of gravity, which obscured the field of view during the separation of the back layer of the tissue gap, prolonging the operation time. Therefore, we tried to use the inverse sequence method for the separation, and the operation time was significantly reduced. In addition, the Huaxi hole 1 broke through the highest point of the breast, allowing the inner and outer lower quadrants of the subcutaneous fat layer to be freed smoothly, and also significantly reduced surgery time. The Huaxi hole 1 is created at the intersection of the areola and the skin. Due to the color difference here, the scar could be hidden and would gradually fade as it slowly heals, not affecting the overall esthetics of the breast.

In terms of cost, the cost varied significantly between patients due to the different types of prosthesis used. For prosthesis combined with mesh, the hospitalization costs for day patients were significantly lower than those for patients in the WEP and WMP groups, which may be related to their shorter hospitalization and lower costs for postoperative care.

During the short-term postoperative follow up at 3 months, no postoperative local recurrence and metastasis were identified, which was also a shortcoming of this study. Our day surgery center only started performing breast endoscopic reconstructive surgery in September 2020, therefore the follow-up time for patients in the DSP group was significantly shorter than for patients in the WEP and WMP groups, which may lead to bias in the statistics of complications. In addition, the completion of the BREAST-Q rating scale was retrospective, which can also result in some bias. In future studies, we will conduct a prospective cohort study of breast endoscopic reconstructive 
surgery in our ward and day surgery center with longterm follow up to explore the safety and efficacy of breast endoscopic reconstructive surgery at a day surgery center.

\section{Conclusions}

The findings of the present study indicate that our selfinitiated endoscopic breast reconstruction surgery is safe and reliable and has high postoperative patient satisfaction when performed at a day surgery center under strict preoperative and postoperative management protocols and mature (the improved procedure was proven to be safe and effective after being performed in the inpatient unit) surgical techniques. After the epidemic is over, this type of surgery can be performed on a regular basis at ambulatory surgery centers.

\section{Acknowledgments}

The authors thank the study participants for their cooperation with our study.

Funding: The present study was funded by the Science and Technology Department of Sichuan Province (No. 2019YFH0146).

\section{Footnote}

Reporting Checklist: The authors have completed the STROBE reporting checklist. Available at https://dx.doi. org/10.21037/gs-21-405

Data Sharing Statement: Available at https://dx.doi. org/10.21037/gs-21-405

Conflicts of Interest: All authors have completed the ICMJE uniform disclosure form (available at https://dx.doi. org/10.21037/gs-21-405). The authors have no conflicts of interest to declare.

Ethical Statement: The authors are accountable for all aspects of the work in ensuring that questions related to the accuracy or integrity of any part of the work are appropriately investigated and resolved. All procedures performed in this study involving human participants were in accordance with the Declaration of Helsinki (as revised in 2013). The study was approved by the ethics board of West China Hospital of Sichuan University (No.: 2021863). Individual consent for this retrospective analysis was waived.

Open Access Statement: This is an Open Access article distributed in accordance with the Creative Commons Attribution-NonCommercial-NoDerivs 4.0 International License (CC BY-NC-ND 4.0), which permits the noncommercial replication and distribution of the article with the strict proviso that no changes or edits are made and the original work is properly cited (including links to both the formal publication through the relevant DOI and the license). See: https://creativecommons.org/licenses/by-nc-nd/4.0/.

\section{References}

1. Halsted WS. I. The Results of Operations for the Cure of Cancer of the Breast Performed at the Johns Hopkins Hospital from June, 1889, to January, 1894. Ann Surg 1894;20:497-555.

2. NARKEVICH FV. Extensive radical mastectomy in cancer of the breast. Zdravookhr Beloruss 1963;9:67-8.

3. Delarue NC, Anderson WD, Starr J. Modified radical mastectomy in the individualized treatment of breast carcinoma. Surg Gynecol Obstet 1969;129:79-88.

4. Thomsen K. Possibilities and limits of breast conserving therapy of carcinoma. Arch Gynakol 1975;219:99-105.

5. Karademas EC, Argyropoulou K, Karvelis S. Psychological symptoms of breast cancer survivors: a comparison with matched healthy controls and the association with cancer-related stress and coping. J Psychosoc Oncol 2007;25:59-74.

6. den Heijer M, Seynaeve C, Timman R, et al. Body image and psychological distress after prophylactic mastectomy and breast reconstruction in genetically predisposed women: a prospective long-term follow-up study. Eur J Cancer 2012;48:1263-8.

7. Lambert M, Mendenhall E, Kim AW, et al. Health system experiences of breast cancer survivors in urban South Africa. Womens Health (Lond) 2020;16:1745506520949419.

8. Askitas N, Tatsiramos K, Verheyden B. Estimating worldwide effects of non-pharmaceutical interventions on COVID-19 incidence and population mobility patterns using a multiple-event study. Sci Rep 2021;11:1972.

9. Zeng C, Zhang J, Li Z, et al. Spatial-Temporal Relationship Between Population Mobility and COVID-19 Outbreaks in South Carolina: Time Series Forecasting Analysis. J Med Internet Res 2021;23:e27045.

10. Dauplat J, Thivat E, Rouanet P, et al. Risk Factors 
Associated With Complications After Unilateral Immediate Breast Reconstruction: A French Prospective Multicenter Study. In Vivo 2021;35:937-45.

11. Kim M, Ku I, Jin US. Diagnosis and management of toxic shock syndrome after breast reconstructive procedures with silicone implants. Arch Plast Surg 2021;48:189-93.

12. Gao GX, Wang ZH, Liu WH, et al. Clinical application of single-port inflatable endoscopic nipple sparing mastectomy with immediate reconstruction using prosthesis implantation. Zhonghua Wai Ke Za Zhi 2021;59:121-6.

13. Toesca A, Peradze N, Galimberti V, et al. Robotic Nipplesparing Mastectomy and Immediate Breast Reconstruction With Implant: First Report of Surgical Technique. Ann Surg 2017;266:e28-30.

14. Toh U, Takenaka M, Iwakuma N, et al. Clinical outcomes of patients after nipple-sparing mastectomy and reconstruction based on the expander/implant technique. Surg Today 2021;51:862-71.

15. Houvenaeghel G, Barrou J, Jauffret C, et al. Robotic Versus Conventional Nipple-Sparing Mastectomy With Immediate Breast Reconstruction. Front Oncol 2021;11:637049.

16. Ozturk CN, Ozturk C, Soucise A, et al. Bilateral immediate two-stage breast reconstruction in patients with unilateral breast cancer: Outcomes analysis and risk assessment. J Plast Reconstr Aesthet Surg 202 1;74:480-5.

17. Dooley WC. Ambulatory mastectomy. Am J Surg 2002;184:545-8; discussion 548-9.

18. Seltzer MH. Partial mastectomy and limited axillary dissection performed as a same day surgical procedure in the treatment of breast cancer. Int Surg 1995;80:79-81.

Cite this article as: Zhou J, Liu X, Feng Y, Li J, Qin X, Huang Y, Yang H, Qiu M, Liu Y, Ma H, Lv Q, Du Z. Breakthrough in breast reconstruction in the context of COVID-19: safety and efficiency of endoscopic breast reconstruction at a day surgery center. Gland Surg 2021;10(8):24772489. doi: $10.21037 / \mathrm{gs}-21-405$
19. Ng EI, Quah GS, Graham S, et al. Immediate prepectoral implant reconstruction using TiLOOP Bra Pocket results in improved patient satisfaction over dual plane reconstruction. ANZ J Surg 2021;91:701-7.

20. Sewart E, Turner NL, Conroy EJ, et al. Patientreported outcomes of immediate implant-based breast reconstruction with and without biological or synthetic mesh. BJS Open 2021;5:zraa063.

21. Lai HW, Chen ST, Mok CW, et al. Robotic versus conventional nipple sparing mastectomy and immediate gel implant breast reconstruction in the management of breast cancer- A case control comparison study with analysis of clinical outcome, medical cost, and patientreported cosmetic results. J Plast Reconstr Aesthet Surg 2020;73:1514-25.

22. Jaberi AA, Zamani F, Nadimi AE, et al. Effect of family presence during teaching rounds on patient's anxiety and satisfaction in cardiac intensive care unit: A doubleblind randomized controlled trial. J Educ Health Promot 2020;9:22.

23. Özaydın AN, Doğan E, Bozdoğan B. Men's Knowledge and Attitudes Towards Breast Cancer: A Descriptive Study. Eur J Breast Health 2020;16:183-91.

24. Visconti G, Franceschini G, Bianchi A, et al. Transaxillary Nipple-Sparing Mastectomy and Direct-to-Implant Breast Reconstruction Using a Simplified Endoscopic Approach: Indications, Cosmetic Outcomes and Technical Refinements. Aesthetic Plast Surg 2020;44:1466-75.

(English Language Editor: R. Scott) 